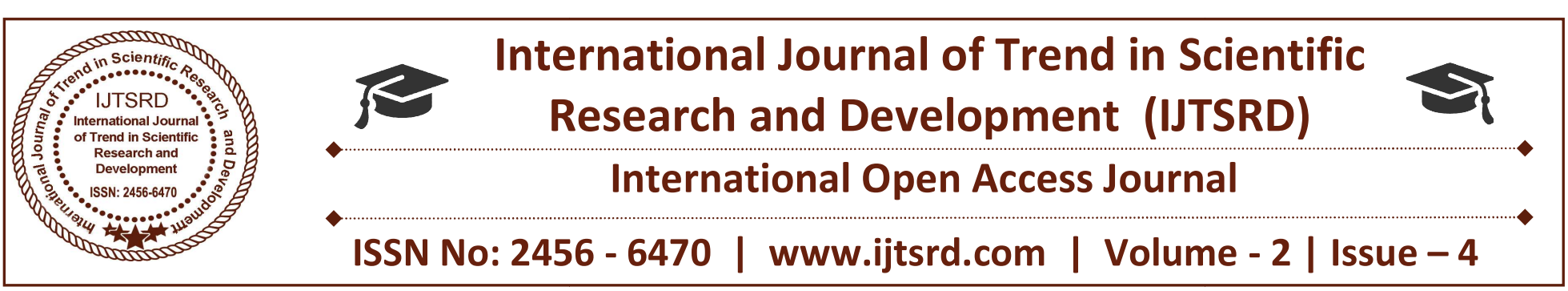

\title{
Corroborative Computational Network Using Specknet in Wireless Routing Algorithm
}

\author{
Dr. Ashok Koujalagi ${ }^{1}$, Thrupti N. $\mathbf{S}^{2}$ \\ ${ }^{1}$ Assistant Professor \& Postdoctoral Researcher, ${ }^{2}$ Assistant Professor \\ ${ }^{1,2}$ P.G Department of Computer Science, Basaveshwar Science College, Bagalkot, Karnataka, India
}

\begin{abstract}
Wireless Sensor Networks (WSN) are networks of low-cost, low-power, multi-functional devices that can be used to monitor physical phenomena. Communication between nodes in a vast network requires routing mechanisms. A Specknet is a programmable computational network of large numbers of minute semiconductor components with integrated sensing, processing and wireless networking capabilities called Specks.

The suitability of the algorithms and the performance of the application are analyzed using several experiments. The results of the experiments indicate that MANET (Mobile Ad-hoc Network) algorithms such as the DSDV (Destination Sequenced Distance Vector) algorithm are candidates for use in certain WSN appli-cations. It is also learnt that the DSDV algorithm, while more responsive when used in small networks, is less scalable than the ZRP (Zone Routing Protocol) algorithm.
\end{abstract}

Keywords: Computer Networking, Networking Algorithms, Network Communication

\section{INTRODUCTION}

In recent years, there have been significant advances in the technology used to build Micro-ElectroMechanical Systems (MEMS), digital electronics, and wireless com-munications. This has enabled the development of low-cost, low-power, multi-functional small sensor nodes that can communicate across short distances .

There has been a lot of research into routing in wireless sensor networks, as sum-marised in and .

Routing in wireless sensor networks is important, as commu-nication between nodes is central to most applications that use them. And routing in wireless sensor networks is different from routing in IP and Mobile Ad-hoc Networks (MANET) because:

- The large numbers of nodes, that make it expensive to construct a global addressing scheme." Furthermore, since several wireless sensor network applications are data-driven, addressing is not as important.

- Most applications require data to flow from several sensor nodes to a given sink.

- There are tight constraints on energy, processing power, and memory.

- In most application scenarios, most wireless sensor network nodes are stationary, except for a few mobile nodes, thereby making topological changes infrequent.

Similarly, according to ad-hoc routing techniques are unsuitable for use in wireless sensor networks because of the larger numbers of nodes, dense deployment, higher failure rates, frequent topology changes, use of the broadcast (as opposed to the point-to-point) communication paradigm, limited power, computational capacities and memories, and infeasibility of using global identifiers.

Several of the objections raised in and are similar, though a few - regarding frequency of topological changes and the use of broadcast paradigms - 
contradict each other.

MANET nodes are highly mobile, and this mobility produces effects similar to node failure, i.e., topological changes. There are also wireless sensor applications where dense deployment and significantly larger numbers of nodes are unnecessary, and global IDs are necessary. However, the available energy and memory capacity is highly limited on wireless sensor networks.

Therefore, in this work, we consider whether and how MANET routing algorithms can be used in wireless sensor networks, and application scenarios of the sort described in the preceding paragraph are discussed.

\section{Characterization of Algorithm}

The primary question that this work set out to answer is whether MANET routing algorithms are suitable for use in wireless sensor networks. This could potentially open up an entirely new class of algorithms for use in wireless sensor networks.

To this end, an exploratory study was carried out, wherein two routing algorithms used in MANETs were implemented on hardware prototypes of Specknets called the Prospeckz IIK.

The Prospeckz IIK is the second generation of a prototype that was developed in order to "enable the rapid development of Specks" as well as applications for Specks. The Prospeckz IIK uses off-the-shelf components, and includes a radio with adjustable signal strengths, an 8-bit microcontroller with 32 Kbytes of FLASH and 2 Kbytes of RAM, and the ability to design analogue circuitries that are software reconfigurable.

The SpeckMAC algorithm was used in the MAC layer. This was done because the and the Zone Routing Protocol (ZRP). The first is a proactive wherein routes to all nodes are stored within each node - while the latter is a hybrid routing algorithm which uses a proactive routing algorithm within defined zones and platform. a reactive algorithm outside these zones.

These algorithms were characterized by considering their memory requirements, power consumption, message delivery latency, and Transmitter/Receiver On-time.

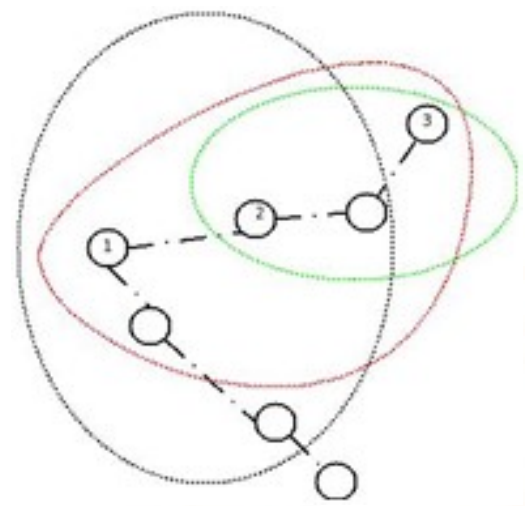

Figure 1.1: A network divided into zones with a zone radius of 2

SpeckMAC algorithm has better power conservation characteristics than other comparable MAC layer algorithms such as the B-MAC.

The two algorithms implemented were the Destination Sequenced Distance Vector (DSDV)

\section{Specknets and the ProSpeckz IIK}

A Speck, "is designed to integrate sensing, processing and wire-less networking capabilities in a minute semiconductor grain". Thousands of Specks, once deployed, are expected to collaborate as "programmable computational networks called Specknets".

As can be seen from these definitions, Specks and Specknets are analogous to the sensor nodes and WSNs aim of the Speckled Computing Project is to enable ubiquitous computing, wherein computation will be performed everywhere, invisible to its users.

Communication between nodes on a Specknet are expected to be a combination of optical and radio communication. 


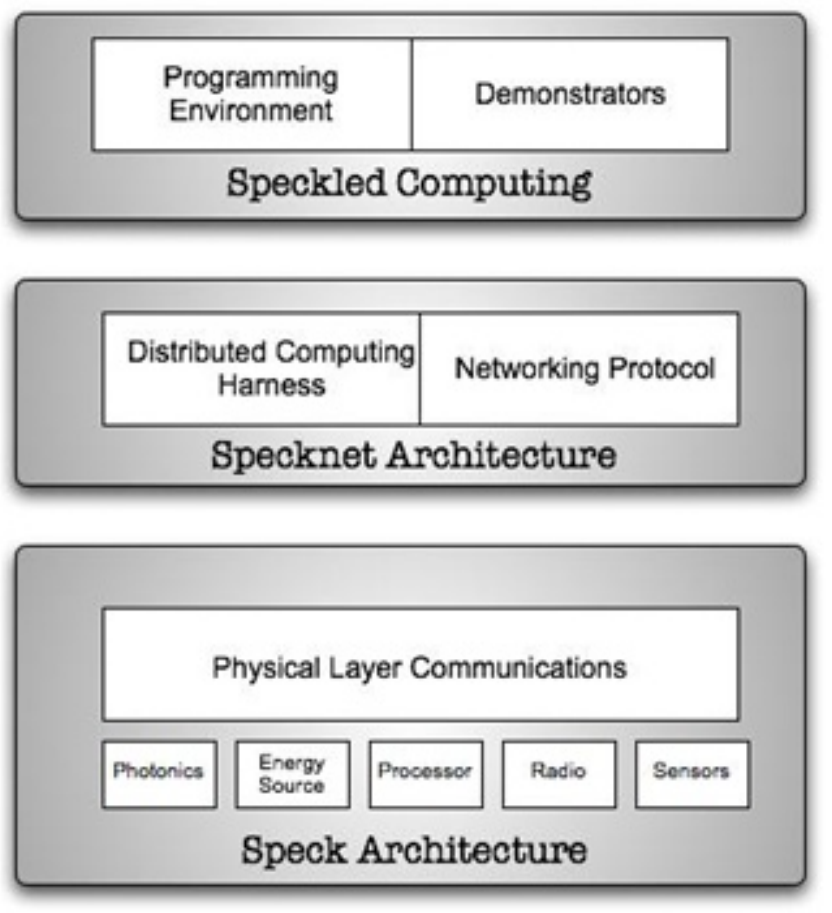

Figure 1.2: Overview of Specknet Architecture

\section{TheProspeckz IIK}

The ProSpeckz (Programmable Specks over Zigbee Radio) is a prototype of Specks developed in order to enable rapid development of Speckled Computing applications. The ProSpeckz, and its successor, the ProSpeckz IIK, have been developed entirely using Commercial off-the-shelf (COTS) components. In the remainder of this section, we provide a short overview of the ProSpeckz IIK

\section{Conclusions and Future Work}

This work presented the implementation and characterisation of a selection of MANET routing algorithms in WSNs. It additionally attempted to explore domains wherein MANET routing algorithms could potentially be applied.

The dissertation began with a review of WSNs, and a detailed discussion of the hardware and software platforms, MAC layer algorithms, and network layer algorithms used (and developed) in this work.

This was followed by an in-depth presentation of the implementation of the DSDV algorithm, an application that builds upon and uses the DSDV algorithm, and the ZRP algorithm. This included descriptions of the position of the algorithm/application in the protocol stack, the primitives used from the lower layers, and the data structures and primitives defined as part of the algorithm/application.

The discussion then moved to the experimental methodology used to characterise the implementations developed herein. The metrics used and the mechanisms used to measure them were presented.

This report then detailed the results obtained upon performing the experiments, and the conclusions derived from these results. The results indicated that the MANET algorithms analysed herein were suitable for use in WSNs by virtue of their power and memory conservation characteristics, and also that there exist application domains where these algorithms may be used.

\section{Bibliography}

1) AKKAYA, K., AND YOUNIS, M. A Survey on Routing Protocols in Wireless Sensor Networks. In Ad-hoc Networks (2005).

2) AKYILDIZ, I. F., SU, W., SANKARASUBRMANIAN, Y., AND CAYIRCI, E. A

3) Survey on Sensor Networks. IEEE Communications Magazine (August 2002), 102114.

4) AL-KARAKI, J. N., AND KAMAL, A. E. Routing Techniques in Wireless Sensor Networks: A Survey. IEEE Wireless Communications (December 2004), 6-28.

5) ARVIND, D. K., AND WONG, K. J. Speckled Computing: Disruptive Technol-ogy for Networked Information Appliances. In IEEE International Symposium on Consumer Electronics (2004), pp. 219-223.

6) AZUMA, R. Tracking requirements for augmented reality. Communications of the ACM (1993), 50-51.

7) BAHL, P., AND PADMANABHAN, V. RADAR: an in-building RF-based user location and tracking system. In Proceedings of the Nineteenth Annual IEEE Joint Conference of the IEEE Computer and Communications Societies (2000), pp. 775-784.

8) BAHL, P., PADMANABHAN, V., AND BALACHANDRAN, A. Enhancements to the RADAR User Location and Tracking System. 
Microsoft Research Technical Report MSR TR2000-12, February 2000.

9) BARNES, M., AND LING, M. The 5CubeOTS. 5th Workshop in Speckled Com-puting, Edinburgh, September 2006.

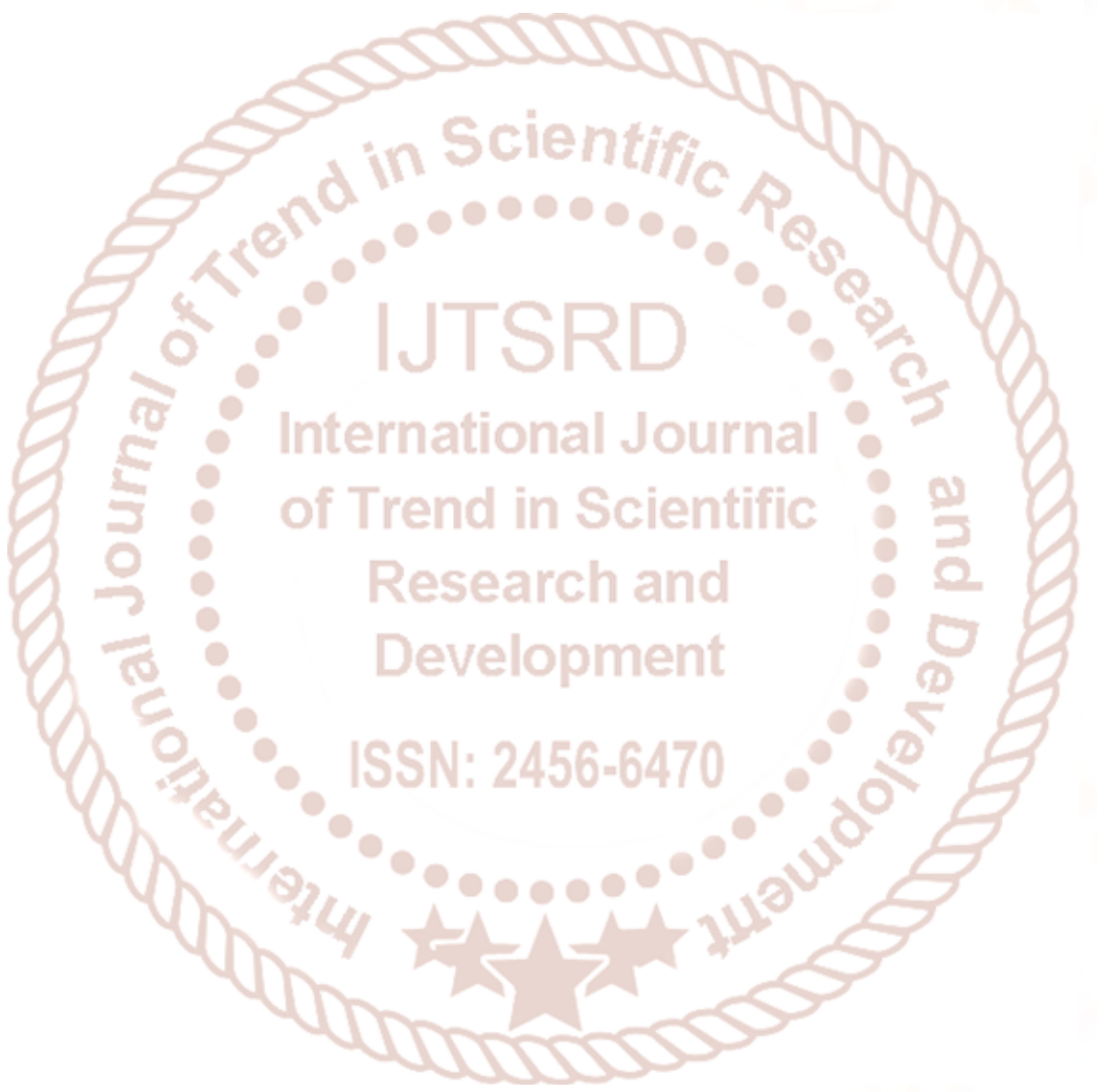

